\title{
VERIFICATION TECHNIQUES FOR SENSITIVITY ANALYSIS AND DESIGN OF CONTROLLERS FOR NONLINEAR DYNAMIC SYSTEMS WITH UNCERTAINTIES
}

\author{
Andreas RAUH* , JOHANnA MINISINI**, EBERHARd P. HOFER ** \\ * Chair of Mechatronics \\ University of Rostock, D-18059 Rostock, Germany \\ e-mail: andreas.rauh@uni-rostock.de \\ ** Institute of Measurement, Control, and Microtechnology \\ University of Ulm, D-89069 Ulm, Germany \\ e-mail: \{johanna.minisini, eberhard.hofer\}@uni-ulm.de
}

\begin{abstract}
Control strategies for nonlinear dynamical systems often make use of special system properties, which are, for example, differential flatness or exact input-output as well as input-to-state linearizability. However, approaches using these properties are unavoidably limited to specific classes of mathematical models. To generalize design procedures and to account for parameter uncertainties as well as modeling errors, an interval arithmetic approach for verified simulation of continuoustime dynamical system models is extended. These extensions are the synthesis, sensitivity analysis, and optimization of open-loop and closed-loop controllers. In addition to the calculation of guaranteed enclosures of the sets of all reachable states, interval arithmetic routines have been developed which verify the controllability and observability of the states of uncertain dynamic systems. Furthermore, they assure asymptotic stability of controlled systems for all possible operating conditions. Based on these results, techniques for trajectory planning can be developed which determine reference signals for linear and nonlinear controllers. For that purpose, limitations of the control variables are taken into account as further constraints. Due to the use of interval techniques, issues of the functionality, robustness, and safety of dynamic systems can be treated in a unified design approach. The presented algorithms are demonstrated for a nonlinear uncertain model of biological wastewater treatment plants.
\end{abstract}

Keywords: interval arithmetic, reachability analysis, observability analysis, robust stability, model-based design of optimal controllers.

\section{Introduction}

Classical approaches to the design of controllers for continuous-time dynamic systems often consist of two stages. In a first stage, a control strategy is determined for both linear and nonlinear dynamical systems under the assumption of a mathematical system model with ideal operating conditions. These ideal conditions are expressed in terms of nominal parameters and nominal initial conditions of the state variables. In most cases, the underlying mathematical models are given by sets of ordinary differential equations (ODEs) and sets of differential-algebraic equations (DAEs). Then, in a second stage, the robustness of the nominal control law with respect to imperfectly known parameters and initial conditions is investigated. Furthermore, it has to be investigated whether the dynamics of the closed-loop control system are influenced significantly by the fact that the design process in Stage 1 is often performed for a reduced, i.e., simplified system model. In contrast to the design of the controller, it has to be evaluated and applied to detailed models which are as close as possible to the real-world operating conditions.

Usually, Monte Carlo simulations or grid-based techniques are applied to quantify the influence of the abovementioned uncertainties (Hammersley and Handscomb, 1964). However, typically no systematic approach is used which rigorously verifies the applicability of control laws under the influence of uncertainties in a guaranteed way from a mathematical point of view. The goal of this paper is to take into account robustness specifications, optimality criteria, criteria for the reachability and observability of states, as well as further specifications result- 
ing from choosing specific structures for control laws and state observers in the earliest possible design stages. The previously mentioned specific structures include, for example, control laws leading to exact state feedback linearization (Marquez, 2003) as well as control laws which directly exploit system properties such as differential flatness (Fliess et al., 1995).

This paper describes the current status of the development of a general-purpose software environment for the design of robust and optimal controllers and state estimators using interval arithmetic. For that purpose, uncertainties in parameters and in initial states will be assumed to be bounded. Up to now, such a general-purpose software tool does not exist. Only for special cases, which are mostly treated in an analytic way, suitable algorithms for controller design are available. In this case, design criteria for robust controllers for linear dynamical systems with uncertain parameters are usually specified using the notions of $\Gamma$-stability or $\mathcal{B}$-stability. Using these notions, robustness can either be specified by regions in the complex domain containing all admissible poles of the closed-loop transfer function ( $\Gamma$-stability) or by specifications of worst-case bounds for the frequency response ( $\mathcal{B}$-stability) (Ackermann et al., 2002; Sienel et al., 1996; Odenthal and Blue, 2000; Bünte, 2000). Then, the parameterization of linear feedback controllers with a predefined structure is possible using the parameter space approach introduced by Ackermann and Kaesbauer (Ackermann et al., 2002).

A special case of $\Gamma$-stability is robust Hurwitzstability which can be verified using Kharitonov's stability criterion. Generalizations leading to the definition of absolute stability enable the design of control laws for dynamical systems which contain sector-bounded static nonlinearities (see, e.g., the Popov criterion). A detailed discussion of these methods and the MATLAB-based software tool PARADISE can be found in (Ackermann et al., 2002).

The above-mentioned software environment only deals with the parameterization of controllers based on robustness requirements. Combinations with optimality criteria and the definition of bounds for the admissible range of control inputs which lead to further constraints on both the parameters of controllers with a predefined structure and to constraints on the controller structure itself are usually not considered. First approaches leading in this direction were proposed in (Rauh and Hofer, 2005; 2009; Rauh et al., 2007d; Rauh et al., 2009). In these articles, interval arithmetic procedures for the design of controllers for linear as well as nonlinear dynamical systems with uncertainties were developed. During the design stages, time domain specifications of robustness as well as optimality criteria are taken into account simultaneously in order to parameterize control laws and to determine appropriate controller structures.
In this paper, the basic functionalities of a general software environment for the design of robust and optimal controllers are described. Furthermore, directions for future research are pointed out. Especially for nonlinear mathematical system models, simplifications of these models are common in controller design. Linearization techniques are used in order to approximate nonlinear dynamics by linear ones. Possibilities for the quantification of the resulting approximation errors by interval parameters are mentioned in Section 2

Also in Section 2, prerequisites for the modeling and design of control systems are summarized. In Section 3, a brief summary of the interval-based simulation environment VALENCIA-IVP (Auer et al., 2008) is given which can be used for a verified analysis of the dynamics of closed-loop control systems with respect to uncertainties of system parameters and variations in controller parameters. Extensions of this simulation tool towards the simulation of sets of DAEs and their relevance with respect to controller design are highlighted in Section 4 In Sections 5 and 6 , relations between computational procedures for verified sensitivity analysis of control laws using VALENCIA-IVP, the simulation of sets of DAEs, and the design of control laws exploiting differential flatness or exact input-output as well as input-to-state linearizability are pointed out. Requirements for robust trajectory planning, computational verification of robust stability, and the design of optimal control laws for systems with uncertainties are discussed in Sections 7.9. In Section 10, computational results of the sensitivity analysis of a nonlinear mathematical model of biological wastewater treatment processes and their applicability with respect to controller design are given. Finally, this paper is concluded with an outlook on future research in Section 11 .

\section{Modeling, analysis, and design of control systems}

In this paper, the modeling, analysis, and design of openloop as well as closed-loop controllers for nonlinear dynamical systems described by sets of ODEs

$$
\dot{x}(t)=f(x(t), p(t), u(t), t)
$$

with $x \in \mathbb{R}^{n_{x}}, p \in \mathbb{R}^{n_{p}}, u \in \mathbb{R}^{n_{u}}$ are discussed. For that purpose, two different scenarios are distinguished.

First, the sensitivity of the controlled systems' trajectories $x(t)$ is analyzed with respect to uncertainties of the initial conditions $x\left(t_{0}\right)$ and the parameters $p(t)$. In this case, either open-loop control laws $u(t)$ or closedloop control laws $u(x(t))$ are assumed to be given. The numerical solution approach is based on calculating guaranteed enclosures of all reachable states using verified ODE solvers such as VALENCIA-IVP. Originally, VALENCIA-IVP has been developed to solve initial 
value problems (IVPs) for ODEs with uncertain parameters $p \in[p ; \bar{p}]$ and uncertain initial conditions $x\left(t_{0}\right) \in$ $\left[\underline{x}\left(t_{0}\right) ; \bar{x}\left(t_{0}\right)\right]$. This functionality is extended by computing partial derivatives of the states $x(t)$ with respect to (uncertain) parameters $p$ using libraries for automatic and algorithmic differentiation.

Second, the basic applications of verified IVP solvers for DAEs are pointed out. In addition to sensitivity analysis, they can be applied to determine open-loop control sequences $u(t)$ numerically such that a specific output variable matches a predefined time response.

The prerequisite for the application of verified solvers for the simulation and controller design of dynamic systems is the availability of suitable mathematical models which have to be detailed enough (without significant simplifications and neglections) such that they can be used to verify control laws. If reduced models are used to carry out the controller design, the influence of simplifications and unavoidable approximation errors should be quantified as precisely as possible. One suitable means for that purpose is the subsumption of the resulting approximation errors in additive, interval-bounded correction terms in the state equations which are taken into account throughout all design stages.

\section{Verified sensitivity analysis using VALENCIA-IVP}

In the following, ODEs $\dot{x}(t)=f(x(t), p, t)$ are considered which, as pointed out in Section 2, describe both open-loop and closed-loop control systems. The vector $p$ consists of all time-invariant system and controller parameters. The differential sensitivities of the solution $x(t)$ with respect to the parameters $p$ are defined by

$$
\dot{s}_{i}(t)=\frac{\partial f(x(t), p, t)}{\partial x} \cdot s_{i}(t)+\frac{\partial f(x(t), p, t)}{\partial p_{i}}
$$

for all $i=1, \ldots, n_{p}$.

The new state vectors $s_{i}(t)$ in (2) are given by

$$
s_{i}(t):=\frac{\partial x(t)}{\partial p_{i}} \in \mathbb{R}^{n_{x}}
$$

with the corresponding initial conditions

$$
s_{i}\left(t_{0}\right)=\frac{\partial x\left(t_{0}, p\right)}{\partial p_{i}} .
$$

If the initial states $x\left(t_{0}\right) \in\left[\underline{x}\left(t_{0}\right) ; \bar{x}\left(t_{0}\right)\right]$ are independent of $p \in[p ; \bar{p}]$, the equality $s_{i}\left(t_{0}\right)=0$ holds. In VALENCIA-IVP, the ODEs (2) do not have to be derived symbolically, since all required partial derivatives with respect to $x$ and $p$ are computed using automatic differentiation provided by FADBAD++ (Bendsten and Stauning, 2007).
For example, defining the linear state equation

$$
\dot{x}(t)=p \cdot x(t)
$$

directly leads to the sensitivity equation

$$
\dot{s}(t)=\frac{\mathrm{d}}{\mathrm{d} t}\left(\frac{\partial x(t)}{\partial p}\right)=p \cdot \frac{\partial x(t)}{\partial p}+x(t) .
$$

This expression does not have to be computed symbolically since it is determined in a straightforward way by VALENCIA-IVP using automatic differentiation.

As for the case of solving an IVP for the ODEs $\dot{x}(t)=f(x(t), p, t)$ using VALENCIA-IVP, guaranteed state enclosures

$$
[x(t)]:=x_{\mathrm{app}}(t)+\left[R_{x}(t)\right]
$$

are determined in a first stage. In (7), the approximate solution $x_{\text {app }}(t)$ for the IVP is determined numerically using a non-verified ODE solver. The guaranteed error bounds $\left[R_{x}(t)\right]$ are calculated iteratively (Rauh et al., $2007 \mathrm{a}$ ). In a second stage, after convergence of the iteration for the interval bounds $[x(t)]$, suitable approximate solutions $s_{i \text {,app }}(t)$ and additional enclosures

$$
\left[s_{i}(t)\right]:=s_{i, \text { app }}(t)+\left[R_{s, i}(t)\right]
$$

with $s_{i, \text { app }}(t) \in \mathbb{R}^{n_{x}}$ and $i=1, \ldots, n_{p}$ are determined for the sensitivities. For both exactly known and uncertain values of $p$ and $x\left(t_{0}\right)$, the intervals $\left[s_{i}(t)\right]$ are determined such that the partial derivatives of all reachable states with respect to all possible $p_{i}$ are included. For time-varying parameters $p(t)$, the sensitivities $s_{i}(t)$ are computed with respect to time-invariant variables $\epsilon_{i} \approx 0$ after substituting $p(t)+\epsilon$ with $\epsilon \in \mathbb{R}^{n_{p}}$ for $p(t)$. The variables $\epsilon_{i}$ are either replaced by the value zero or by time-invariant intervals containing the value zero.

The calculation of the differential sensitivities $s_{i}(t)$ using a verified ODE solver provides useful information for the design of controllers. Guaranteed bounds of sensitivities of the state variables can be obtained for uncertain parameters $p \in[p]$ using a single evaluation of the state equations even for non-monotonic relations between the parameters $p$ and the state variables $x$. Techniques for the reduction of overestimation are available which combine consistency tests and exponential state enclosures to avoid the growth of the diameters of the state enclosures, especially for asymptotically stable systems (Rauh $e t a l$., 2007b).

\section{Application of VALENCIA-IVP to DAEs}

In previous work, VALENCIA-IVP has been extended to determine guaranteed state enclosures also for DAEs (Rauh et al., 2007b). In the following, semi-explicit 
DAEs

$$
\begin{aligned}
\dot{x}(t) & =f(x(t), y(t), t) & \text { with } f: D \mapsto \mathbb{R}^{n_{x}}, \\
0 & =g(x(t), y(t), t) & \text { with } g: D \mapsto \mathbb{R}^{n_{y}},
\end{aligned}
$$

$D \subset \mathbb{R}^{n_{x}} \times \mathbb{R}^{x_{y}} \times \mathbb{R}^{1}$, with the consistent initial conditions $x\left(t_{0}\right)$ and $y\left(t_{0}\right)$ are evaluated. These DAEs may further depend on uncertain parameters $p$. To simplify the notation in Sections 4 and 5, the dependence on $p$ is not explicitly denoted. However, all properties are also applicable to systems with $p \in[\underline{p} ; \bar{p}], \underline{p}<\bar{p}$.

For verified DAE solvers, there are two important applications. First, as for verified simulation of ODEs, the influence of uncertainties can be analyzed by calculating guaranteed state enclosures if consistent initial conditions are given for $x\left(t_{0}\right)$ and $y\left(t_{0}\right)$. Second, open-loop control strategies are determined such that the system's output signal matches a predefined time response. This task is often referred to as the inverse control problem. For example, for nonlinear exactly input-to-state linearizable sets of ODEs, this task can be solved symbolically by expressing $u(t)$ (as one component of $y(t)$ in (9) and (10)) in terms of the state variables of the exactly linearized system. However, numerical design approaches based on interval analysis provide more flexibility since uncertainties and robustness requirements can be taken into account directly. For that purpose, sets of ODEs and DAEs are extended by time-dependent algebraic constraints to specify the desired output. The corresponding solution provides the desired control and an enclosure of all reachable states.

\section{Analysis of reachability and observability and exact feedback linearization}

In this section, nonlinear input-affine dynamical systems

$$
\dot{x}(t)=f(x(t))+g(x(t)) \cdot u(t)
$$

with output equations

$$
y(t)=h(x(t))
$$

are considered. Non-input-affine systems $\dot{x}=f(x, u)$ can be transformed using artificial control inputs $\tilde{u}$ according to $\dot{x}=f(x, u), \dot{u}=\tilde{u}, y=h(x)$.

\subsection{Analysis of reachability for nonlinear dynamical} systems. Prerequisites for the implementation of controllers are the reachability and observability of states. The corresponding criteria for nonlinear systems are generalizations of Kalman's criteria for state controllability and observability for linear systems. To derive these criteria, techniques from differential geometry have to be applied (Isidori, 1989). For nonlinear dynamical systems the question whether a specific state is reachable with a given input has to be analyzed to design for example a controller with input-to-state linearization.

The Lie brackets of $f(x)$ and $g(x)$ which are defined by $[f(x), g(x)]=\frac{\partial g(x)}{\partial x} f(x)-\frac{\partial f(x)}{\partial x} g(x)$ describe a new direction that can be reached by a control for a given dynamical system (11). After applying these Lie brackets successively one gets all possible reachable directions in the state-space. Thus with the help of $P_{0}(x)=g(x)$, $P_{1}(x)=[f(x), g(x)]$, and $P_{k}(x)=\left[f(x), P_{k-1}(x)\right]$, $k=2, \ldots, n_{x}-1$, the state-dependent reachability matrix

$$
P(x)=\left[\begin{array}{llll}
P_{0}(x) & P_{1}(x) & \ldots & P_{n_{x}-1}(x)
\end{array}\right]
$$

is determined. This matrix is evaluated for the intervals $[x]$ of the state variables. Using a verified LU-decomposition of interval matrices, the rank of $P(x) \in \mathbb{R}^{n_{x} \times n_{x} n_{u}}$ is determined. It corresponds to the dimension of the reachable manifold of the dynamical system for all $x \in[x]$, where $[x]$ is identified using interval techniques.

\subsection{Analysis of observability for nonlinear dynam-} ical systems. The observability matrix is defined with the help of the Lie derivatives $L_{f}^{i} h(x)=L_{f}\left(L_{f}^{i-1} h(x)\right)$ with $L_{f}^{0} h(x)=h(x)$ and $L_{f} h(x)=\frac{\partial}{\partial x} h(x) \cdot f(x)$ which provide information about the variation of the output $h(x)$ along the vector field $f(x)$. Successive application of the Lie derivatives leads to the observability matrix

$$
Q(x)=\left[\begin{array}{llll}
Q_{0}^{T}(x) & Q_{1}^{T}(x) & \ldots & Q_{n_{x}-1}^{T}(x)
\end{array}\right]^{T}
$$

with $Q_{i}(x)=\frac{\partial}{\partial x} L_{f}^{i} h(x)$ for all $i=0, \ldots, n_{x}-1$. Again, using a verified LU-decomposition of interval matrices, the rank of $Q(x) \in \mathbb{R}^{n_{x} n_{y} \times n_{x}}$ has to be determined, which is equal to a sufficient criterion for the dimension of the observable manifold of the dynamical system. Routines for path and trajectory planning have to make sure that both matrices $P(x)$ and $Q(x)$ have full rank $n_{x}$ to guarantee reachability and observability in a verified sense even if system parameters are not known exactly.

In Appendix, a sample program is shown which can be used to compute the observability matrix $Q(x)$ using automatic differentiation in $\mathrm{C}++$. The observability matrix $Q(x)$ is computed using the interval arithmetic library PROFIL/BIAS (Keil, 2007) and FADBAD++ (Bendsten and Stauning, 2007) providing functionalities of automatic differentiation.

The application of libraries for automatic differentiation for the computation of Lie derivatives was presented in (Röbenack, 2002). However, the author did not use the results in an interval-based framework for model-based controller design. The routines for the computation of Lie derivatives are based on determining Taylor coefficients of the dynamic system model. These Lie derivatives are also necessary to identify hidden constraints in the simulation 
of DAEs. In this case, the time derivatives $\mathrm{d}^{i} g / \mathrm{d} t^{i}=0$, $i \geq 1$, of (10) are required.

Note that the rank criteria for reachability and observability only provide local information within an interval box $[x]$. In addition, unique identifiability of states is required if non-measured internal variables are to be reconstructed using state estimators. For a simple example with indistinguishable states, consider $\dot{x}=u$, $y_{1}=\cos (x), y_{2}=\sin (x)$ for which the states $\tilde{x}$ and $\tilde{x}+2 \pi k, k \in \mathbb{Z}$, are indistinguishable by the measured outputs $y_{1}$ and $y_{2}$. For further references about the reachability and observability of nonlinear dynamical systems, see (Hermann and Krener, 1977; Sontag, 1998).

Interval techniques can be easily applied to check if a state vector $x$ can be reconstructed uniquely using the measured data $y$. For that purpose, enclosures of all states within a priori given domains are determined using interval Newton methods such that they are consistent with $y$ and a finite number of its time derivatives. Unique identifiability is guaranteed if a single consistent state vector exists in the domain under consideration.

\subsection{Controller design: Input-to-state linearization.} The goal of controller design is to convert the input-affine dynamical system into a set of linear ODEs

$$
\dot{z}(t)=A \cdot z(t)+B \cdot w(t) \quad \text { with } \quad y(t)=C \cdot z(t)
$$

using a coordinate transformation $z=\tau(x): D \subset$ $\mathbb{R}^{n_{x}} \mapsto \mathbb{R}^{n_{x}}$ and a control law $u=r(x)+W(x) \cdot w$ for exact input-to-state linearization. Let $\delta_{i}$ be the relative degree of the output $y_{i}, i=1, \ldots, m$, i.e., the smallest order of the derivative $\mathrm{d}^{\delta_{i}} y / \mathrm{d} t^{\delta_{i}}$ which explicitly depends on $u$. Then, the state transformation

$$
z=\tau(x)=\left[\begin{array}{lllll}
\tau_{1}^{1}(x) & \ldots & \tau_{1}^{\delta_{1}}(x) & \tau_{2}^{1}(x) & \ldots
\end{array}\right]^{T}
$$

can be computed using the Lie derivatives $\tau_{i}^{r_{i}}=$ $L_{f}^{r_{i}-1} h_{i}(x), r_{i}=1, \ldots, \delta_{i}$. given by

The feedback control law $u=r(x)+W(x) \cdot w$ is

$$
r(x)=-D^{-1}(x) \varphi(x) \text { and } W(x)=D^{-1}(x) .
$$

In (17), the vector

$$
\varphi(x)=\left[\begin{array}{llll}
\varphi_{1}(x) & \varphi_{2}(x) & \ldots & \varphi_{m}(x)
\end{array}\right]_{x(t)=\tau^{-1}(z)}^{T}
$$

is defined by $\varphi_{i}(x)=L_{f}^{\delta_{i}} h_{i}(x)$ for all $i=1, \ldots, m$. Furthermore, the decoupling matrix $D(x)$ is computed by

$$
D(x)=\left[\begin{array}{ccc}
L_{g_{1}} L_{f}^{\delta_{1}-1} h_{1}(x) & \cdots & L_{g_{m}} L_{f}^{\delta_{1}-1} h_{1}(x) \\
L_{g_{1}} L_{f}^{\delta_{2}-1} h_{2}(x) & \cdots & L_{g_{m}} L_{f}^{\delta_{2}-1} h_{2}(x) \\
\vdots & \vdots & \vdots \\
L_{g_{1}} L_{f}^{\delta_{m}-1} h_{m}(x) & \cdots & L_{g_{m}} L_{f}^{\delta_{m}-1} h_{m}(x)
\end{array}\right] .
$$

Linear feedback controllers can be designed for 15 if $\operatorname{rank}\{D(x)\}=n_{x}$ and $\delta=\delta_{1}+\ldots+\delta_{m}=n_{x}$ hold for all desired states $x(t)$, all possible parameter values $p$, and uncertainties of $x\left(t_{0}\right)$. These prerequisites can be verified by interval evaluation of $D(x)$ for all reachable states. The required derivatives in (18) and (19) have already been computed by automatic differentiation for the reachability and observability matrices (13) and (14), respectively.

\section{Relations to controller design for differentially flat systems}

A dynamic system $\dot{x}(t)=f(x(t), u(t))$ is called differentially flat if the so-called flat outputs

$$
y=y\left(x, u, \dot{u}, \ldots, u^{(\alpha)}\right)
$$

exist such that

(i) all system states $x$ as well as all inputs $u$ can be expressed as functions of the flat outputs and their time derivatives according to

$$
x=x\left(y, \dot{y}, \ldots, y^{(\beta)}\right)
$$

and

$$
u=u\left(y, \dot{y}, \ldots, y^{(\beta+1)}\right)
$$

(ii) the flat outputs $y$ are differentially independent, i.e., they are not coupled by differential equations.

Differential flatness is a generalization of input-tostate linearizability, because the flat outputs $y$ need not be the physical outputs of the dynamical system. Furthermore, it results from (i) that (ii) is equivalent to $\operatorname{dim}(u)=\operatorname{dim}(y)$. For the special case of linear systems, differential flatness is equivalent to controllability (Fliess et al., 1995).

\section{Robust trajectory planning}

As shown in the preceding sections, the reachability as well as the observability of states are two basic requirements for the design of most control strategies for dynamic systems. In order to fulfill the design requirements, it is necessary on the one hand to influence the state variables of a dynamic system in a deterministic way (guarantee of reachability). On the other hand, those quantities that are required for state feedback controllers which are either not measurable directly or for which sensors are avoided due to reasons of reliability or cost-effectiveness have to be guaranteed to be reconstructable by modelbased observer techniques (guarantee of observability). An application for which this is crucial is discussed in Section 10 
However, often further restrictions have to be incorporated during controller design. The previously discussed criteria for the analysis of reachability and observability do not take into account limitations of control variables and states which are usually relevant. In general, there are two possibilities how such limitations can be considered by model-based approaches in the design of controllers.

First, the so-called sigmoid functions such as arctangent or hyperbolic tangent can be applied. For example, if a control input $u$ is limited to the range $[\underline{u} ; \bar{u}]$, a suitable limiting function is

$$
u:=\left(\frac{1}{\pi} \arctan (\tilde{u})+\frac{1}{2}\right) \cdot(\bar{u}-\underline{u})+\underline{u},
$$

where the reachability of states now has to be verified with respect to the artificial input $\tilde{u}$.

Second, verified simulation of time responses using interval techniques is applicable to check whether control sequences are guaranteed to stay within their prescribed bounds and to determine strategies how they have to be modified to meet the restrictions, see Section 9

This directly leads to the problem of path and trajectory planning. For given initial and final points of trajectories in the design of tracking controllers, states for which reachability and/or observability are lost have to be avoided by suitable reference inputs of the closedloop control system. Furthermore, trajectories have to be planned such that limitations of states and control variables are preserved. Typical state constraints in path planning for applications in robotics are obstacles as well as safety critical operating conditions which are considered, for example, in (Pepy et al., 2008).

Finally, requirements for the validity of a specific design approach such as the regularity of the decoupling matrix $D(x)$ in Section 5 have to be guaranteed for all states along planned trajectories.

\section{Stability analysis for nonlinear control systems with uncertainties}

For nonlinear dynamical systems, Lyapunov functions provide a suitable means for the numerical and-in special cases - analytical proof of asymptotic stability. In general, both nominal system models as well as system models with parameter uncertainties can be considered.

An equilibrium $x_{\infty}$ of a nonlinear dynamical system (1) is stable, if a continuously differentiable function $V(x, p): D \mapsto \mathbb{R}$ with

$$
\begin{array}{ll}
V(x, p)=0 & \text { for } x=x_{\infty} \\
V(x, p)>0 & \text { for } x \neq x_{\infty} \\
\dot{V}(x, p) \leq 0 & \text { for } x \neq x_{\infty}
\end{array}
$$

exists. The equilibrium $x_{\infty}$ of the dynamical system (1) is characterized by

$$
f\left(x_{\infty}, p, u, t\right)=0,
$$

where $u=u\left(x_{\infty}\right)=u_{\infty}=$ const and $p=p_{\infty}=$ const hold. The validity of the stability criterion (24) must be guaranteed in a neighborhood of the equilibrium $x_{\infty}$. A dynamical system is globally stable if (24) holds for all $x \in \mathbb{R}^{n_{x}}$.

If the derivative with respect to time of the function $V(\cdot)$ in (24) can be proven to be negative definite instead of negative semi-definite along the trajectories of the dynamical system, it is asymptotically stable in a neighborhood of the equilibrium (Marquez, 2003; Khalil, 2002). Furthermore, using these conditions, regions in the statespace can often be identified which belong to the region of attraction of asymptotically stable equilibria (Marquez, 2003).

Using interval arithmetic techniques, two different approaches for the verification of stability properties of nonlinear dynamical systems can be distinguished:

- stability analysis based on interval evaluation of the above-mentioned Lyapunov functions, and

- tests for the convergence of guaranteed enclosures of the sets of all reachable states over time towards an equilibrium.

The prerequisite for both approaches is the calculation of a guaranteed enclosure $\left[x_{\infty}\right]$ of the unique equilibrium $x_{\infty}$ using interval Newton techniques. If the dynamical system model depends on interval parameters $p \in[p]$, the equilibrium is usually parameter dependent. Then, the set of all possible equilibria has to be included within the box $\left[x_{\infty}\right]$.

For the sake of simplicity, only time-invariant dynamical systems are considered in the following. However, time-varying characteristics can be dealt with after suitable modification of the algorithms.

8.1. Stability analysis using interval evaluation of Lyapunov functions. The following description of interval-based stability analysis using Lyapunov functions is based on (Delanoue, 2006). After the computation of the interval enclosure $\left[x_{\infty}\right]$, a double-valued approximate solution $\tilde{x}_{\infty} \in\left[x_{\infty}\right]$ is chosen. Then, an approximation $A$ of the system's Jacobian is determined for $\tilde{x}_{\infty}$, i.e.,

$$
A:=\left.\frac{\partial f}{\partial x}\right|_{x=\tilde{x}_{\infty}} .
$$

Using this matrix, the Lyapunov equation

$$
A^{T} P+P A=-I
$$


of the linearized system is solved for the symmetric matrix $P$. If $P$ is positive definite, i.e., if the linearized system can be proven to be asymptotically stable, an estimate for the region of attraction of an asymptotically stable equilibrium of the original nonlinear system can be determined.

For that purpose, an interval box $\left[x_{0}\right]$ for which $\left[x_{\infty}\right] \subset\left[x_{0}\right]$ holds is assumed. Additionally, this box must not contain further equilibria. Therefore, typically the initialization of the interval Newton iteration used to determine $\left[x_{\infty}\right]$ is chosen.

To analyze the stability of the dynamical system, the quadratic Lyapunov function

$$
V(x, p)=\left(x-x_{\infty}\right)^{T} \cdot P \cdot\left(x-x_{\infty}\right)
$$

with $P$ determined in 27 is used. For the time derivative of this Lyapunov function, the properties

$$
\left.\dot{V}(x, p)\right|_{x=x_{\infty}}=0 \quad \text { and }\left.\quad \frac{\partial \dot{V}(x, p)}{\partial x}\right|_{x=x_{\infty}}=0
$$

hold. Then the Hessian

$$
H:=-\frac{\partial^{2} \dot{V}(x, p)}{\partial x^{2}}
$$

has to be shown to be positive definite for all $x \in\left[x_{0}\right]$. This can be done using a procedure described by Rohn in (Rohn, 1994). A symmetric interval matrix $[H]=$ $\left\{H \mid H_{c}-\Delta \leq H \leq H_{c}+\Delta\right\}$ with $H_{c}=\frac{1}{2}(\underline{H}+\bar{H})$ and $\Delta=\frac{1}{2}(\bar{H}-\underline{H})$ is positive definite if the following $2^{n_{x}-1}$ point matrices $H_{z}=H_{-z}$ are positive definite. The matrices $H_{z}$ are defined according to $H_{z}:=$ $H_{c}-T_{z} \cdot \Delta \cdot T_{z}$ with $T_{z}:=\operatorname{diag}\{(z)\}$. The vector $z$ has to be replaced by all possible combinations of the components $z_{i}= \pm 1, i=1, \ldots, n_{x}$. Thus, $H_{z}=H_{-z}$ holds.

As shown in (Delanoue, 2006), the interval box $[x]$ with center in $\left[x_{\infty}\right]$ and radius

$$
\sqrt{n_{x} \frac{\lambda_{\min }}{\lambda_{\max }}} d\left(\left[x_{\infty}\right],\left[x_{0}\right]\right)
$$

certainly belongs to the region of attraction of an asymptotically stable equilibrium $x_{\infty}$. In (31), $\lambda_{\min }$ and $\lambda_{\max }$ are the minimum and maximum eigenvalues of $P$, respectively. Furthermore, $d$ is a function defined on $\mathbb{I R}^{n_{x}} \times$ $\mathbb{I} \mathbb{R}^{n_{x}}$ with

$$
d:([x],[y]) \mapsto \sup \{r \in \mathbb{R} \mid B(r,[x]) \subset[y]\},
$$

an interval box $[x] \subset \mathbb{R}^{n_{x}}$, and $B(r,[x])$ denoting the set

$$
\left\{x \in \mathbb{R}^{n_{x}} \mid \min _{a \in[x]}\|a-x\|<r\right\} .
$$

Example 1. Consider the nonlinear dynamical system

$$
\dot{x}(t)=-x(t)+x(t)^{3} .
$$

Using interval Newton methods such as verifynlss in the MATLAB toolbox INTLAB (Rump, 2007), tight enclosures of the three equilibria $x_{\infty}=0$ and $x_{\infty}= \pm 1$ can be verified. Considering the equilibrium $x_{\infty}=0$, the evaluation of the Lyapunov equation (27) leads to $P=0.5$. Due to the positivity of $P$ and due to the requirement for positivity of $H=2-12 x^{2}$ in a neighborhood of $x_{\infty}=0$, the evaluation of (31) leads to the interval $[-0.4082 ; 0.4082]$ which is guaranteed to be contained in the region of attraction of the asymptotically stable equilibrium $x_{\infty}=0$.

Example 2. Now, the nonlinear system

$$
\begin{aligned}
& \dot{x}_{1}(t)=3 x_{2}(t) \\
& \dot{x}_{2}(t)=-5 x_{1}(t)+x_{1}(t)^{3}-2 x_{2}(t)
\end{aligned}
$$

is considered.

In this example, asymptotic stability of the equilibrium $x_{\infty}=\left[\begin{array}{ll}0 & 0\end{array}\right]^{T}$ is verified. According to Subsection 8.1 positive definiteness of the symmetric matrices

$$
H_{1}:=\left[\begin{array}{ll}
\underline{H}_{11} & \underline{H}_{12} \\
\underline{H}_{12} & \underline{H}_{22}
\end{array}\right] \text { and } H_{2}:=\left[\begin{array}{ll}
\underline{H}_{11} & \bar{H}_{12} \\
\bar{H}_{12} & \underline{H}_{22}
\end{array}\right]
$$

has to be proven. These matrices are determined with the help of (26)-30). To check definiteness properties, the eigenvalues of $H_{1}$ and $H_{2}$ are determined with the interval routine verifyeig provided by INTLAB to take into account the effect of rounding errors. Finally, the equilibrium under consideration can be shown to be asymptotically stable. Additionally, the interval

$$
[x]=\left[\begin{array}{l}
{[-0.496 ; 0.496]} \\
{[-0.496 ; 0.496]}
\end{array}\right]
$$

is guaranteed to be contained in its region of attraction.

8.2. Stability analysis using verified integration of ODEs. In addition to the stability analysis using Lyapunov functions, the computation of guaranteed state enclosures can also be used to determine interval boxes included in the regions of attraction of stable equilibria. For that purpose, the set of all reachable states is computed, starting from an interval box $\left[x_{0}\right]$ at a point of time $t=t_{1}$, see the left hand side of Fig. 1

If it can be shown that the set of reachable states at a point of time $t_{2}>t_{1}$ is included completely in the interior of $\left[x_{0}\right]$, the stability of the dynamical system has been proven under the condition that the mathematical system 

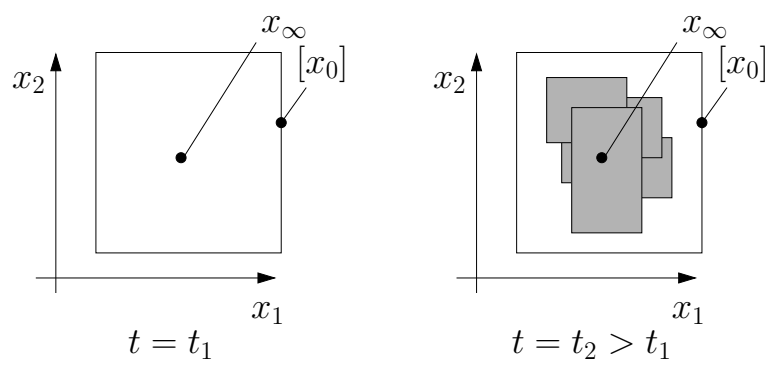

Fig. 1. Stability analysis of nonlinear dynamical systems using verified integration of ODEs.

model does not explicitly depend on time. On the right hand side of Fig. 1, a typical result is shown. In order to identify stability, the splitting of interval enclosures into smaller subdomains is usually inevitable. The result of the propagation of these subintervals is illustrated by grey boxes in Fig 1

Note that due to overestimation, which often occurs in interval evaluation of dynamical system models, both approaches for the verification of stability properties are only sufficient criteria. If one of these methods fails to verify (asymptotic) stability, either the attempt to prove instability or the exploitation of further techniques for reduction of overestimation - and thus for computation of tighter interval bounds - are suitable further steps.

\section{Integration in an interval arithmetic framework for the design of robust and optimal controllers}

The interval arithmetic techniques introduced so far in this paper can be integrated in a systematic approach for the computation of optimal control strategies. In this framework for the computation of control laws, verified evaluation of integral performance indices

$$
\begin{aligned}
J= & f_{t_{f}}\left(x\left(t_{f}\right), p\left(t_{f}\right), t_{f}\right) \\
& +\int_{0}^{t_{f}} f_{0}(x(t), p(t), u(t), t) \mathrm{d} t
\end{aligned}
$$

is carried out. The performance indices $J$ to be minimized generally consist of two additive terms. The term $f_{t_{f}}\left(x\left(t_{f}\right), p\left(t_{f}\right), t_{f}\right)$ corresponds to terminal costs at the prescribed final point of time $t_{f}$ if final states $x\left(t_{f}\right)$ are not specified exactly. The integrand $f_{0}(x(t), p(t), u(t), t)$ quantifies both deviations of the current states from the desired trajectories of the state variables and the required control effort $u(t)$.

A general framework for interval arithmetic structure and parameter optimization for dynamic systems with both nominal and uncertain parameters was presented (Rauh and Hofer, 2009; Rauh et al., 2007d). Using the definition of optimality for uncertain systems introduced therein, a control strategy is optimal if it leads to the smallest upper bound of the performance index for all possible $p \in[p]$.

The techniques presented in Sections 3,8 provide further information that is included in this framework for controller design. First, the results of reachability, observability, and stability analysis of the open-loop control system make clear whether the desired control task can be solved at all for a specific dynamic system model under consideration of the available actuators and available sensors. Before the initialization of the routine for structure and parameter optimization, a choice between these two different tasks is possible. Furthermore, also combinations between structure optimization for some of the control inputs and classical design approaches for feedback control of other control inputs are possible at this stage if multi-input systems are handled.

For structure optimization, the result of the optimization algorithm is a control sequence which directly aims at the minimization of the performance index. In the case of parameter optimization, a control law $u(x)$ with free parameters is given. This control law is parameterized again such that the performance index $J$ is minimized.

In Fig. 2, an algorithm for the calculation of both optimal control strategies in structure optimization problems as well as parameter optimization problems is summarized. Since it relies on verified integration of the set of state equations for different controller parameterizations, it can be easily parallelized. For that purpose, different independent tasks are defined. In the case of structure optimization, usually independent optimization problems are solved in separate tasks which correspond to control strategies with different numbers of switchings between piecewise constant control inputs $u(t)$, where their number is denoted by $N_{1}, \ldots, N_{m}$. Alternatively, piecewise linear control strategies can be determined, where the variation rates of the control input $u$ are constant between two subsequent switching points. In the case of parameter optimization, the number of piecewise constant controls is $N_{1}=\cdots=N_{m}=1$ in all tasks. This means that the free parameters in the control law $u(x)$ to be determined are formally treated as time-invariant inputs which are calculated with exactly the same routine that is used for structure optimization. However, the assignment of the subintervals of the controller parameters to the different tasks, which is related to the number of switchings of the control input in the structure optimization problem, now has to be carried out in a slightly different way. All candidates for controller parameterizations for which inadmissibility (due to the violation of state constraints or due to the violation of constraints for the range of the control inputs $u$ after the parameterization of a predefined controller structure) or non-optimality (due to the existence of a control sequence with a smaller upper bound $\bar{J}^{*}$ of 
Reachability and observability analysis of open-loop control system with uncertainties

Stability analysis of open-loop control system

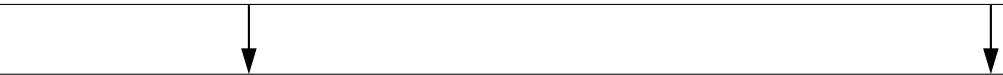

Coice between parameterization of predefined control structure or structure optimization

Combination of structure optimization and feedback control



(1) Extension to robust trajectory planning by exclusion of inadmissible controls and inadmissible reference signals

Fig. 2. Parallelized implementation of the interval arithmetic procedure for the calculation of optimal and robust control strategies. 
the performance index for all $p \in[p]$ ) has not yet been shown are distributed equally to the available processors using $m$ tasks.

In addition to optimality criteria, also sensitivity measures for the system states $x(t)$ as well as the performance index $J$ with respect to the uncertain parameters can be taken into account to find a specific robust controller parameterization in a systematic way.

After the termination of the optimization procedure, again the stability of the closed-loop control system can be analyzed. Performing a verified sensitivity analysis using VALENCIA-IVP helps to choose a specific controller parameterization if several admissible candidates for the controller structure or controller parameterization with similar values of the underlying cost function exist.

Finally, information about regions of attraction of asymptotically stable equilibria allows identifying the set of admissible operating conditions. These can be expressed in terms of sets of admissible initial states. Furthermore, the maximum admissible deviations of the actual trajectories of a dynamical system from the desired ones can be quantified such that the stability of the complete system is ensured also in the case of disturbances. This task as well as extensions for path and trajectory planning which help to fulfill design criteria for specific approaches for controller design (such as exact feedback linearization) will be investigated in greater detail in future research.

\section{Application scenario: A biological wastewater treatment process}

10.1. Modeling and controller design. Basic applications of verified techniques for controller design and sensitivity analysis are demonstrated for the subsystem model of biological wastewater treatment depicted in Fig. 3 . This subsystem model is a simplification of the Activated Sludge Model No. 1 of the International Water Association (Henze et al., 2002). In contrast to the complete Activated Sludge Model, the reduction of nitrogen fractions is not considered in this subsystem model.

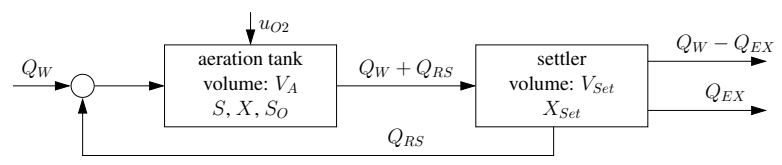

Fig. 3. Block diagram of a simplified biological wastewater treatment process.

The concentration $S$ of biodegradable organic substrate is reduced by heterotrophic bacteria with the concentration $X$ under external oxygen supply with the flow rate $u_{O 2}$. The concentration of dissolved oxygen in the aeration tank is denoted by $S_{O}$. The bacteria concentration in the settler, which is modeled as a perfect separator of sludge and purified water, is denoted by $X_{\text {Set }}$. A portion of the activated sludge is fed back into the aeration tank with the flow rate $Q_{R S}$ of return sludge. The excess sludge $Q_{E X}$ is removed from the process.

According to (Rauh, Auer and Hofer, 2007), this process is described by the nonlinear ODEs

$$
\begin{aligned}
\dot{S}= & \frac{Q_{W}}{V_{A}}\left(S_{W}-S\right)-\mu\left(S, S_{O}\right) \frac{1}{Y} X, \\
\dot{X}= & -\frac{Q_{W}}{V_{A}} X+\frac{Q_{R S, \text { nom }}}{V_{A}}\left(X_{\text {Set }}-X\right) \\
& +\left(\mu\left(S, S_{O}\right)-b\right) X \\
& +\frac{Q_{R S, \text { nom }}}{V_{A}}\left(X_{\text {Set }}-X\right) \Delta Q_{R S}, \\
\dot{S}_{O}= & \frac{Q_{W}}{V_{A}}\left(S_{O W}-S_{O}\right)-\mu\left(S, S_{O}\right) \frac{1-Y}{Y} X \\
& +\frac{\rho_{O 2}}{V_{A}}\left(1-\frac{S_{O}}{S_{O, \text { sat }}}\right) u_{O 2}, \\
\dot{X}_{\text {Set }}= & \frac{Q_{W}+Q_{R S, \text { nom }}}{V_{\text {Set }}}-\frac{Q_{E X}+Q_{R S}}{V_{\text {Set }}} X_{\text {Set }} \\
& +\frac{Q_{R S, \text { nom }}}{V_{\text {Set }}} X \Delta Q_{R S},
\end{aligned}
$$

where the nonlinear growth rate of substrate consuming bacteria is modeled by the Monod kinetics

$$
\mu\left(S, S_{O}\right)=\hat{\mu}_{H} \frac{S}{S+K_{S}} \frac{S_{O}}{S_{O}+K_{O S}} .
$$

A complete list of all parameters can be found in (Rauh et $a l ., 2007 \mathrm{c})$. In contrast to the above-mentioned references, two control inputs $u_{O 2}$ and $\Delta Q_{R S}$ are considered in the state equations 39.

In the following, the design and parameterization of controllers which compensate variations of states caused by parameter uncertainties rely on the adaptation of the available control variables based on the results of a sensitivity analysis. In addition to the oxygen input rate $u_{O 2}$, related to the desired oxygen concentration $\hat{S}_{O}$ via (41), the flow rate $Q_{R S}=Q_{R S, \text { nom }} \cdot\left(1+\Delta Q_{R S}\right)$ of return sludge is considered as a second control variable in this paper. As usual for biological wastewater treatment plants, the flow rate $Q_{R S}$ is assumed to be time-invariant. Additionally, it is assumed that the sum of the flow rates $Q_{R S}+Q_{E X}$ does not change if $Q_{R S}$ is adjusted.

Available measured data are provided by a sensor for the concentration $S_{O}$ of dissolved oxygen and a sensor determining a signal that is proportional to the sum of the concentrations $S$ and $X$ via the measurement of the attenuation of light intensity in the aeration tank.

Assuming a constant oxygen concentration $S_{O}=$ $\hat{S}_{O}=$ const, i.e., $\dot{S}_{O}=0$, the corresponding feedforward 
control for the oxygen input rate $u_{\mathrm{O} 2}$ is defined by

$$
\begin{aligned}
u_{O 2}= & \frac{V_{A}}{\rho_{O 2}} \frac{S_{0, \mathrm{sat}}}{S_{0, \mathrm{sat}}-\hat{S}_{0}}\left(\mu\left(S, \hat{S}_{O}\right) \frac{1-Y}{Y} X\right. \\
& \left.-\frac{Q_{W}}{V_{A}}\left(S_{O W}-\hat{S}_{O}\right)\right) .
\end{aligned}
$$

10.2. Verified analysis of the observability and reachability of states. Using the criterion for observability analysis of nonlinear systems and evaluating it for interval boxes containing the trajectories of all reachable states with $S_{O}=\hat{S}_{O}$ and the initial conditions and parameters from (Rauh et al., 2007c), complete state observability can be verified for all $\hat{\mu}_{H} \in[0.9 ; 1.1] \hat{\mu}_{H, \text { nom. }}$. Although the observability of the system can also be proven for tight enclosures of the system states if $S_{O}$ is considered as the only measured state variable, additional use of $S+X$ significantly improves the robustness of state observability. This fact is even more advantageous in practical application since sensors in wastewater treatment processes are subject to rough environmental conditions leading to nonnegligible measurement uncertainties and reduced reliability.

In contrast to observability analysis, the reachability of states can only be verified for small uncertainties of $\hat{\mu}_{H}$ and tight enclosures of the state trajectories. For larger intervals, the reachability matrix contains elements for which its full rank cannot be proven.

Since a flatness-based control strategy was implemented for this system after minor simplifications in (Aschemann et al., 2006) and since the following sensitivity-based approach shows a possibility regarding how to influence the substrate concentration $S$ in a desired way, this shortcoming is of minor importance for the application considered. In general, the problem to decide whether the reachability matrix has full rank and whether a possible loss of this property is only caused by overestimation resulting from interval evaluation of the Lie brackets can be solved by systematic subdivisions of the interval enclosures of the reachable states. Afterwards, the reachability criterion has to be evaluated for each of the subintervals.

10.3. Sensitivity analysis for the adaptation of control variables. The differential sensitivities of the state variables $S, X$, and $X_{\text {Set }}$ with respect to variations $\Delta S_{O}$ and $\Delta Q_{R S}$ of the control variables and with respect to variations $\Delta \hat{\mu}_{H}$ of the growth rate of substrate consuming bacteria with

$$
\begin{aligned}
S_{O} & :=\hat{S}_{O} \cdot\left(1+\Delta S_{O}\right), \\
\hat{\mu}_{H} & :=\hat{\mu}_{H, \mathrm{nom}} \cdot\left(1+\Delta \hat{\mu}_{H}\right), \\
Q_{R S} & :=Q_{R S, \mathrm{nom}} \cdot\left(1+\Delta Q_{R S}\right), \\
Q_{E X} & :=Q_{E X, \mathrm{nom}}-Q_{R S, \mathrm{nom}} \cdot \Delta Q_{R S}
\end{aligned}
$$

provide useful information for the parameterization of $\Delta S_{O}$ and $\Delta Q_{R S}$ to influence the state variables for all parameters $\hat{\mu}_{H} \in[0.9 ; 1.1] \hat{\mu}_{H, \text { nom. }}$.

For $\hat{S}_{O}=3.5 \cdot 10^{-3} \mathrm{~kg} / \mathrm{m}^{3}$, these sensitivities were computed using VALENCIA-IVP for three different growth rates of substrate consuming bacteria as well as for its complete parameter interval $\hat{\mu}_{H} \in[0.9 ; 1.1] \hat{\mu}_{H, \text { nom }}$. The results of these simulations are shown in Fig. 4, where each curve represents the guaranteed enclosure for one of the examined values of $\hat{\mu}_{H}$. Note that the diameters of the resulting enclosures computed for the point values $0.9 \hat{\mu}_{H, \text { nom }}, 1.0 \hat{\mu}_{H, \text { nom }}$, and $1.1 \hat{\mu}_{H, \text { nom }}$ are below the resolution of these graphs. For the complete parameter interval, the interval bounds which contain the results for evaluation with point values are clearly visible in Fig. 4

In this case, the evaluation was aborted at the point of time at which the interval enclosures of the state variables had become negative due to overestimation. This overestimation arises since the state enclosures have been described by a single interval box. Special techniques for the reduction of overestimation which make use of subdivision strategies are not necessary in this scenario since the required modifications of $\hat{S}_{O}$ and $\Delta Q_{R S}$ to influence $S(t)$ in a deterministic way can already be derived from the results shown in Fig. 4

Furthermore, negative states are guaranteed to be caused by overestimation since the analysis of (39) shows that the variation rates of all state variables are guaranteed to remain non-negative for physically reasonable, positive parameters and positive initial states as soon as one of the state variables reaches the zero.

According to the results of sensitivity analysis in


duced rate of the reduction of the substrate concentration $S$. Furthermore, they also cause reduced bacteria concentrations $X$. This can be compensated by increasing $\hat{S}_{O}$ and reducing $Q_{R S}$. These measures are necessary to meet legal performance requirements for wastewater treatment plants which are specified, for example, in (Office for Official Publications of the European Communities, 2003).

The effects that are achievable by modifications of $\hat{S}_{O}$ and $Q_{R S}$ at a fixed point of time can be estimated by

$$
\Delta S(t) \approx \frac{\partial S(t)}{\partial \Delta S_{O}} \cdot \Delta S_{O}+\frac{\partial S(t)}{\partial \Delta Q_{R S}} \cdot \Delta Q_{R S} .
$$

This estimate makes use of the differential sensitivities of the substrate concentration $S(t)$ which are depicted in Figs. 4(a) and 4(e). Due to physical restrictions, only values $\Delta S_{O}$ are admissible which lead to oxygen concentrations $\hat{S}_{O}$ that are smaller than the saturation concentration $S_{O \text {, sat }}=5.3 \cdot 10^{-3} \mathrm{~kg} / \mathrm{m}^{3}$. Similarly, only positive values are admissible for $Q_{R S}$.

Thus, for the compensation of the influence of a reduced growth rate $\hat{\mu}_{H}$, the choice $\Delta S_{O}=0.50$ and $\Delta Q_{R S}=-0.90$ is investigated. 


$$
\begin{aligned}
-\hat{\mu}_{H} & =0.9 \hat{\mu}_{H, n o m} \\
---\hat{\mu}_{H} & =1.0 \hat{\mu}_{H, n o m} \\
-\cdots \hat{\mu}_{H} & =1.1 \hat{\mu}_{H, n o m} \\
-\hat{\mu}_{H} & \in[0.9 ; 1.1] \hat{\mu}_{H, n o m}
\end{aligned}
$$

(a)

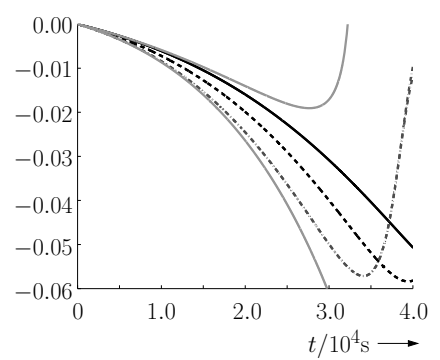

(d)

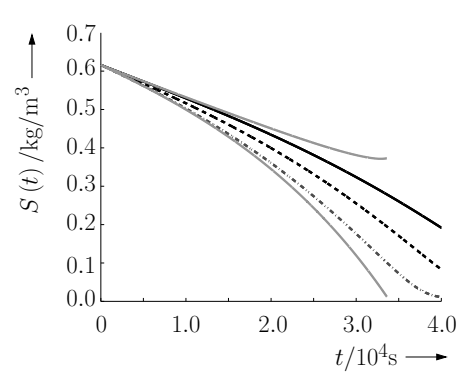

(b)

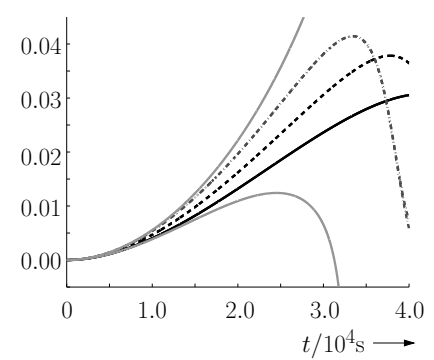

(e)

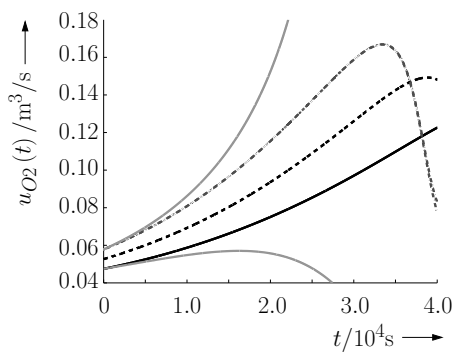

(c)



(f)

Fig. 4. Sensitivity analysis of a biological wastewater treatment process for $t \in[0 ; 40,000] \mathrm{s}$ : (a) legend, (b) substrate concentration $S(t)$, (c) oxygen input rate $u_{O 2}(t)$, (d) sensitivity $\frac{\partial S(t)}{\partial \Delta S_{O}}$, (e) sensitivity $\frac{\partial S(t)}{\partial \Delta Q_{R S}}$, (f) sensitivity $\frac{\partial S(t)}{\partial \Delta \hat{\mu}_{H}}$.

Figure 5(a) shows that larger reductions in the substrate concentration $S(t)$ can be achieved over the complete time horizon by the adaptations $\Delta S_{O}$ and $\Delta Q_{R S}$. The drawback of the increased oxygen concentration $\hat{S}_{O}$ and the reduced flow rate of return sludge $Q_{R S}$ is that the oxygen input rate increases by a factor of approximately 30, see Fig. 5(b). Further improvements are possible if time-varying control sequences $\Delta Q_{R S}(t)$ are considered. However, such strategies are hard to implement since most wastewater treatment plants are operating with a constant ratio of the flow rates of both return and excess sludge. Furthermore, by means of optimization techniques, the parameters $\Delta S_{O}$ and $\Delta Q_{R S}$ can be adjusted such that further influence factors, for example, cost functions describing the effort for oxygen supply are taken into account.

Stability analysis of the equilibria of (39) is not performed here. In general, the above-mentioned time domain analysis provides more information for controller design of this wastewater treatment process since uncertainties and variations in the amount and concentrations in the inflow prevent the operation of the plant in its steady state.

\section{Conclusions and outlook}

In this paper, an overview of the existing interval arithmetic routines which are applicable to the design and verification of control systems has been given.

In future research, further general-purpose strategies will be developed to make use of the results obtained by interval evaluation of the criteria in Section 5 to generate reference signals for controllers such that reachabil- ity and observability are guaranteed. Additionally, routines for the analysis of asymptotic stability of closed-loop controllers will be implemented if the relative degree $\delta$ in the design of feedback linearizing control laws is smaller than $n_{x}$. In this case, instabilities of the internal dynamics have to be detected and avoided in a guaranteed way.

Results obtained for the design of controllers will be extended to implement software tools which are furthermore also applicable to observer design. Finally, combinations with feedforward control strategies, for example, control sequences determined by the approach based on DAEs mentioned in Section 4 will be investigated. With this approach, alternatives for the design of flatness-based control laws will be developed for systems which are either not differentially flat or for which flatness-based approaches lead to complicated analytical expressions. In these cases, this approach is assumed to provide suitable approximations of control laws within prescribed tolerances from the desired time response of the systems' outputs.

\section{Acknowledgment}

The authors would like to thank the reviewers for their valuable suggestions for improving of this paper.

Parts of this work were performed while Andreas Rauh was with the Institute of Measurement, Control, and Microtechnology, University of Ulm, Germany.

\section{References}

Ackermann, J., Blue, P., Bünte, T., Güvenc, L., Kaesbauer, D., Kordt, M., Muhler, M. and Odenthal, D. (2002). Ro- 




(a)

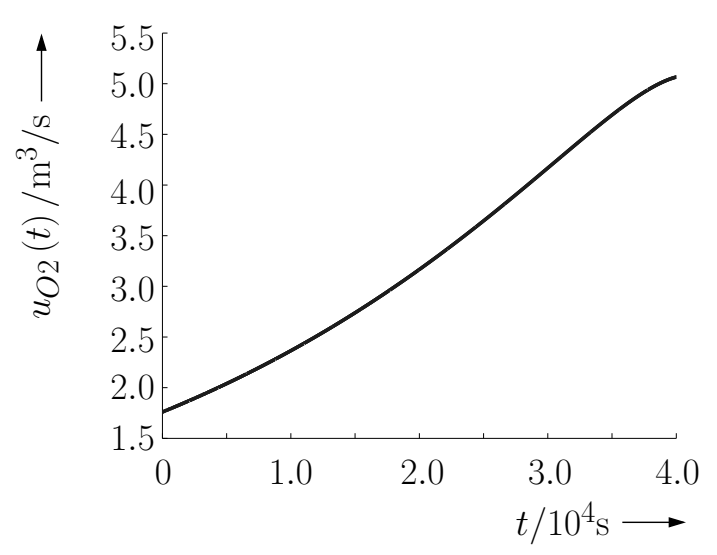

(b)

Fig. 5. Substrate concentration and the oxygen input rate of the wastewater treatment process for the time interval $t \in[0 ; 40,000] \mathrm{s}$ after the adaptation of the control variables: (a) substrate concentration $S(t)$, (b) oxygen input rate after the adaptation of the concentration $\hat{S}_{O}$ and the flow rate $Q_{R S}$.

bust Control: The Parameter Space Approach, 2nd Edn., Springer-Verlag, London.

Aschemann, H., Rauh, A., Kletting, M. and Hofer, E. P. (2006). Flatness-based control of a simplified wastewater treatment plant, Proceedings of the IEEE International Conference on Control Applications CCA 2006, Munich, Germany, pp. 2243-2248.

Auer, E., Rauh, A., Hofer, E. P. and Luther, W. (2008). Validated modeling of mechanical systems with SMARTMOBILE: Improvement of performance by VALENCIA-IVP, Proceedings of the Dagstuhl Seminar 06021: Reliable Implementation of Real Number Algorithms. Theory and Practice, Dagstuhl, Germany, Lecture Notes in Computer Science, Vol. 5045, Springer-Verlag, Berlin/Heidelberg, pp. 1-27.

Bendsten, C. and Stauning, O. (2007). FADBAD++, Version 2.1, available at: http://www. fadbad.com
Bünte, T. (2000). Mapping of Nyquist/Popov theta-stability margins into parameter space, Proceedings of the 3rd IFAC Symposium on Robust Control Design, Prague, Czech Republic.

Delanoue, N. (2006). Algoritmes numériques pour l'analyse topologique-Analyse par intervalles et théorie des graphes, Ph.D. thesis, École Doctorale d'Angers, Angers, (in French).

Fliess, M., Lévine, J., Martin, P. and Rouchon, P. (1995). Flatness and defect of nonlinear systems: Introductory theory and examples, International Journal of Control 61(6): 1327-1361.

Hammersley, J. M. and Handscomb, D. C. (1964). Monte-Carlo Methods, John Wiley \& Sons, New York, NY.

Henze, M., Harremoës, P., Arvin, E. and la Cour Jansen, J. (2002). Wastewater Treatment, 3rd Edn., Springer-Verlag, Berlin.

Hermann, R. and Krener, A. J. (1977). Nonlinear controllability and observability, IEEE Transactions on Automatic Control 22(5): 728-740.

Isidori, A. (1989). Nonlinear Control Systems, 2nd Edn., Springer-Verlag, Berlin.

Keil, C. (2007). ProfiL/BIAS, Version 2.0.4, Available at: www.ti3.tu-harburg.de/keil/profil/

Khalil, H. K. (2002). Nonlinear Systems, 3rd Edn., PrenticeHall, Upper Saddle River, NJ.

Marquez, H. J. (2003). Nonlinear Control Systems, John Wiley \& Sons, Inc., Hoboken, NJ.

Odenthal, D. and Blue, P. (2000). Mapping of frequency response magnitude specifications into parameter space, Proceedings of the 3rd IFAC Symposium on Robust Control Design, Prague, Czech Republic.

Office for Official Publications of the European Communities (2003). Council Directive of 21 May 1991 Concerning Urban Waste Water Treatment (91/271/EEC), Available at: http://ec.europa.eu/environment/water /water-urbanwaste/directiv.html

Pepy, R., Kieffer, M. and Walter, E. (2008). Reliable robust path planner, Proceedings of the IEEE/RSJ International Conference on Intelligent Robots and Systems (IROS), Nice, France, pp. 1655-1660.

Rauh, A., Auer, E. and Hofer, E. P. (2007a). VALEnCIA-IVP: A comparison with other initial value problem solvers, Proceedings of the 12th GAMM-IMACS International Symposium on Scientific Computing, Computer Arithmetic, and Validated Numerics SCAN 2006, Duisburg, Germany, IEEE Computer Society, Los Alamitos, CA, (on CDROM).

Rauh, A., Auer, E., Minisini, J. and Hofer, E. P. (2007b). Extensions of VALENCIA-IVP for reduction of overestimation, for simulation of differential algebraic systems, and for dynamical optimization, PAMM 7(1): 1023001-1023002.

Rauh, A., Kletting, M., Aschemann, H. and Hofer, E. P. (2007c). Reduction of overestimation in interval arithmetic simulation of biological wastewater treatment processes, 
Journal of Computational and Applied Mathematics 199(2): 207-212.

Rauh, A., Minisini, J. and Hofer, E. P. (2007d). Interval techniques for design of optimal and robust control strategies, Proceedings of the 12th GAMM-IMACS International Symposium on Scientific Computing, Computer Arithmetic, and Validated Numerics SCAN 2006, Duisburg, Germany, IEEE Computer Society, Los Alamitos, CA, (on CDROM).

Rauh, A. and Hofer, E. P. (2005). Interval arithmetic optimization techniques for uncertain discrete-time systems, Proceedings of the 13th International Workshop on Dynamics and Control, Modeling and Control of Autonomous Decision Support Based Systems, Wiesensteig, Germany, Shaker Verlag, Aachen, pp. 141-148.

Rauh, A. and Hofer, E. P. (2009). Interval methods for optimal control, Proceedings of the 47th Workshop on Variational Analysis and Aerospace Engineering 2007, Erice, Italy, Springer-Verlag, New York, NY, pp. 397-418.

Rauh, A., Minisini, J. and Hofer, E. P. (2009). Towards the development of an interval arithmetic environment for validated computer-aided design and verification of systems in control engineering, Proceedings of the Dagstuhl Seminar 08021: Numerical Validation in Current Hardware Architectures, Dagstuhl, Germany, Lecture Notes in Computer Science, Vol. 5492, Springer-Verlag, Berlin/Heidelberg, pp. $175-188$.

Röbenack, K. (2002). On the efficient computation of higher order maps $a d_{f}^{k} g(x)$ using Taylor arithmetic and the Campbell-Baker-Hausdorff formula, in A. Zinober and D. Owens (Eds.), Nonlinear and Adaptive Control, Lecture Notes in Control and Information Science, Vol. 281, Springer, Berlin/Heidelberg, pp. 327-336.

Rohn, J. (1994). Positive definiteness and stability of interval matrices, SIAM Journal on Matrix Analysis and Applications 15(1): 175-184.

Rump, S. M. (2007). InTLAB, Version 5.4, available at: http://www.ti3.tu-harburg.de/ rump/ intlab/

Sienel, W., Bünte, T. and Ackermann, J. (1996). PARADISEParametric robust analysis and design interactive software environment: A MATLAB-based robust control toolbox, Proceedings of the 1996 IEEE International Symposium on Computer-Aided Control System Design, Dearborn, MI, USA, pp. 380-385.

Sontag, E. D. (1998). Mathematical Control TheoryDeterministic Finite Dimensional Systems, SpringerVerlag, New York, NY.

\section{Appendix}

Implementation of observability analysis in $\mathrm{C}++$. In the following, an excerpt of a $\mathrm{C}++$ program for observability analysis is given, see also Section 5.2 In this program, the state equations

$$
\begin{aligned}
& \dot{x}_{1}(t)=x_{2}(t) \cdot p, \\
& \dot{x}_{2}(t)=x_{1}(t) \cdot x_{2}(t)
\end{aligned}
$$

with

$$
y(t)=x_{1}^{2}(t)
$$

and $x_{1} \in[-1 ; 1], x_{2} \in[2 ; 2]$, and $p=1.0$ are considered for the construction of the matrix $Q(x)$ in (14).

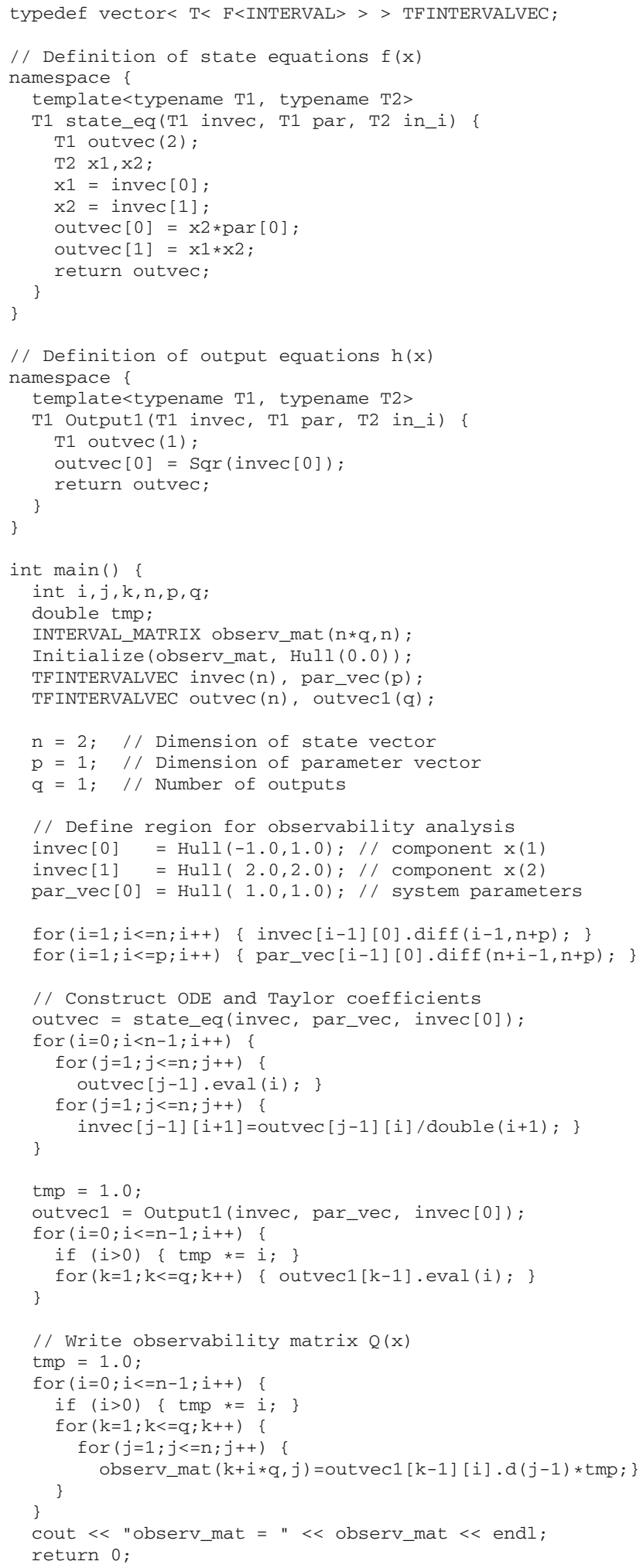




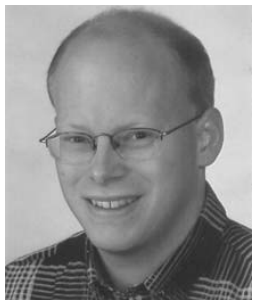

Andreas Rauh received his diploma degree in electrical engineering and information technology from Technische Universität München, Munich, Germany, in 2001 and his Ph.D. degree (Dr.-Ing.) from the University of Ulm, Germany, in 2008. His research interests include state and parameter estimation for stochastic and set-valued uncertainties, verified simulation of nonlinear uncertain systems, nonlinear, robust, and optimal control, interval methods for ordinary differential equations as well as differentialalgebraic systems. Currently, he is with the Chair of Mechatronics, University of Rostock, Germany, as a post-doctoral researcher.

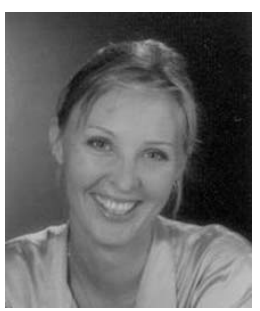

Johanna Minisini received her B.Sc. with honours from the University of New South Wales, Australia, in 1994 and her diploma degree in mathematics from Universität Regensburg, Germany, in 1997. Her research interests cover systematic approaches for the analysis and design of controllers and state estimators for nonlinear dynamical systems. Currently, she is with the Institute of Measurement, Control, and Microtechnology, University of Ulm,

Germany, where she is working on her Ph.D. thesis.

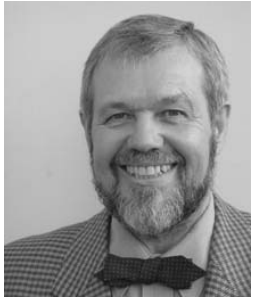

Eberhard P. Hofer is a retired director of the Institute of Measurement, Control, and $\mathrm{Mi}$ crotechnology at the University of Ulm, Germany. He received his doctorate in optimal control and his habilitation qualification in engineering cybernetics from the University of Stuttgart. He has worked for industry (in Germany and the USA) as a project manager in process control and software engineering. He has been a visiting professor at IBM, UC Berkeley, Waseda University in Tokyo, Kobe University, and the Japan Advanced Institute of Science and Technology. His research activities cover systems theory, modeling, control, optimization, robotics, and interval methods with applications to technical and biomedical systems. For his achievements he has received numerous research awards in applied sciences.

Received: 22 September 2008 Revised: 15 December 2008 